\title{
Comparison of Patient Satisfaction between Subcutaneous Immunotherapy and Sublingual Immunotherapy in Allergic Rhinitis
}

\author{
Nopphawan Longchuphon, Su Jin Kim, Kun Hee Lee, Joong Saeng Cho, and Sung Wan Kim \\ Department of Otorhinolaryngology, School of Medicine, Kyung Hee University, Seoul, Korea
}

피하 면역요법과 설하 면역요법의 환자 만족도 비교

Nopphawan Longchuphon · 김수진 · 이건희 · 조중생 · 김성완

경희대학교 의과대학 이비인후과학교실

Received January 5,2015

Revised August 17,2015

Accepted August 27, 2015

Address for correspondence

Sung Wan Kim, MD, PhD

Department of Otorhinolaryngology,

School of Medicine,

Kyung Hee University,

23 Kyungheedae-ro,

Dongdaemun-gu,

Seoul 02447, Korea

Tel $+82-2-958-8474$

Fax $+82-2-958-8470$

E-mail drkimsw@hanmail.net
Background and Objectives Comparative clinical studies of sublingual and subcutaneous treatments have yielded heterogeneous results. In this study, we compared clinical outcomes in patients allergic to house dust mites (HDMs) that received at least 1 year of subcutaneous immunotherapy (SCIT) or sublingual immunotherapy (SLIT).

Subjects and Method The present study included 120 patients with HDM allergic rhinitis, 54 patients in the SCIT group and 66 patients in the SLIT group. Each patient was asked to answer a set of questionnaire before starting immunotherapy. The questionnaires included the rhinoconjunctivitis quality of life questionnaire (RQLQ) and a patient satisfaction questionnaire. The patients were asked to answer the questions after undergoing at least 1 year of immunotherapy.

Results RQLQ scores collected after immunotherapy in both SCIT and SLIT groups were decreased significantly. However, the SCIT group had a significantly higher decrease in RQLQ scores for non-nose/eye symptoms than the SLIT group $(p$-value $=0.015)$. There were no differences in other satisfaction scores between the two groups. SCIT improved clinical symptoms faster than SLIT did within 6 months and 1 year $(p$-value $=0.011, p$-value $=0.045$ respectively).

Conclusion SCIT is more effective than SLIT in improving non-nose/eye symptoms in patients with allergic rhinitis. SCIT may relieve the symptoms faster than SLIT.

Korean J Otorhinolaryngol-Head Neck Surg 2016;59(2):120-4

Key Words Allergic rhinitis · Immunotherapy · Patient satisfaction ·

Rhinoconjunctivitis quality of life questionnaire.

\section{Introduction}

Because allergic disorders can be a significant burden to patients, efforts have been directed to understanding the mechanisms of allergic disorders to develop more rational therapeutic interventions. Im Immunotherapy aims to prevent the immunological reaction of allergy sufferers to specific allergens. Immunotherapy is indicated for the treatment of allergic rhinitis and allergic asthma and may prevent development of asthma in patients with allergic rhinitis. ${ }^{2,3)}$ The current standard therapy is subcutaneous administration of clinically relevant allergens for several months, building up to eventual monthly injections lasting typically 3 to 5 years. ${ }^{2-4)}$

However, subcutaneous immunotherapy (SCIT) has several disadvantages such as a higher risk of anaphylaxis and must be performed only in a clinician's office with adequate personnel 
and equipment to handle anaphylactic events and low compliance in young patients due to pain. ${ }^{2-5)}$ Therefore, other allergen administration methods such as intranasal, oral and sublingual routes have been developed to solve these problems. Among these methods, sublingual immunotherapy (SLIT) has been preferred. $^{4-7)}$

Comparative clinical studies of sublingual and subcutaneous treatments yielded heterogeneous results. Some studies showed symptoms and medication scores were reduced by approximately $50 \%$ in both SLIT and SCIT groups ${ }^{8)}$ and found disease severity was reduced to one-third in the SCIT group and half in the SLIT group compared with placebo-treated patients. ${ }^{9)}$ But the other study reported SLIT and SCIT had the same efficacy and no differences in medical usage in patients with allergic rhinitis to house dust mites (HDMs) who received immunotherapy for at least 6 months. ${ }^{10,11)}$ However, no comparative clinical studies have been conducted regarding SLIT and SCIT in Korea. In the present study we compared clinical outcomes and satisfaction of patients allergic to HDMs after at least 1 year of SCIT or SLIT.

\section{Subjects and Method}

Patients with allergic rhinitis to HDMs (age range from 5-75 years) who decided to receive immunotherapy were divided into either the SCIT group or SLIT group depending on the method of immunotherapy chosen. SCIT was performed by liquid type vaccine (Hollister-Stier, Spokane, WA, USA), SLIT was performed by Staloral ${ }^{\circledR}$ (Stallergenes S.A. Antony, France) which contains sodium chloride (0.059 g), glycerol (0.58 g), purified water, and 300 index of reactivity HDM in $1 \mathrm{~mL}$.

Each patient was asked to answer the questionnaires before starting immunotherapy. The questionnaires used for the study were rhinoconjuntivitis quality of life (RQLQ). The RQLQ has
28 questions in 7 domains (activity limitation, sleep problems, nose symptoms, eye symptoms, non-nose/eye symptoms, practical problems, and emotional function). There are 3 'patientspecific' questions in the activity domain which allow patients to select 3 activities in which they are most limited by their rhinoconjunctivitis. The overall RQLQ score is the mean of all 28 responses and the individual domain scores are the means of the items in those domains.

We compared the time interval when the patients experienced symptom improvement for the first time after starting immunotherapy. Also we compared hospital visit frequency, physician visiting time, adverse effects, medication use, health-care costs and overall satisfaction between SCIT and SLIT groups.

The patients were asked to answer the questionnaires after receiving at least 1 year of immunotherapy. The parents or caretakers of patients under 15 years of age were asked to answer the questionnaires.

All statistical analyses were performed using SPSS, version 11.5 software (SPSS Inc., Chicago, IL, USA). The Student t test, chi square test were used to compare the demographic data between the 2 groups. When comparing patients' satisfaction, Wilcoxon rank-sum test or linear by linear association was used to compare the decreased RQLQ scores or the differences in satisfaction scores from the questionnaire between the 2 groups. The Wilcoxon signed-rank test was used to compare RQLQ scores before and after immunotherapy in each group. $p$ values $<0.05$ were considered to indicate statistical significance for all analyses.

\section{Results}

The study included 120 patients with allergic rhinitis to HDMs, 54 patients in the SCIT group and 66 patients in the SLIT group. There were no differences in demographic data

Table 1. Demographic characteristics and symptom scores before immunotherapy

\begin{tabular}{|c|c|c|c|}
\hline Demographic data & SCIT & SLIT & $p$-value \\
\hline$\overline{\text { Gender }(M / F)}$ & $40 / 14$ & $38 / 28$ & 0.059 \\
\hline Age (years) & $24.33(6-71)$ & $20.92(5-58)$ & 0.511 \\
\hline Duration (months) & $28.11(12-132)$ & $22.77(12-78)$ & 0.051 \\
\hline Activity limitation & $2.02 \pm 0.78$ & $1.84 \pm 1.09$ & 0.082 \\
\hline Sleep problems & $1.69 \pm 1.22$ & $1.46 \pm 1.18$ & 0.284 \\
\hline Nose symptoms & $2.45 \pm 0.73$ & $2.25 \pm 1.02$ & 0.247 \\
\hline Eye symptoms & $0.99 \pm 0.81$ & $0.93 \pm 0.92$ & 0.446 \\
\hline Non-nose/eye symptoms & $1.01 \pm 0.55$ & $1.22 \pm 0.93$ & 0.341 \\
\hline Practical problems & $0.96 \pm 0.78$ & $0.90 \pm 1.04$ & 0.325 \\
\hline Emotional function & $0.76 \pm 0.66$ & $0.91 \pm 0.87$ & 0.512 \\
\hline
\end{tabular}

SCIT: subcutaneous immunotherapy, SLIT: sublingual immunotherapy 
Table 2. Comparisons of RQLQ scores in the SCIT group before and after receiving at least 1 year of immunotherapy

\begin{tabular}{lccc}
\hline \multicolumn{1}{c}{ RQLQ } & Before SCIT & After SCIT & p-value* \\
\hline Activity limitation & $2.02 \pm 0.78$ & $1.30 \pm 0.79$ & $<0.001$ \\
Sleep problems & $1.69 \pm 1.22$ & $0.82 \pm 0.86$ & $<0.001$ \\
Nose symptoms & $2.45 \pm 0.73$ & $1.59 \pm 0.71$ & $<0.001$ \\
Eye symptoms & $0.99 \pm 0.81$ & $0.61 \pm 0.58$ & $<0.001$ \\
Non-nose/eye symptoms & $1.01 \pm 0.55$ & $0.61 \pm 0.44$ & $<0.001$ \\
Practical problems & $0.96 \pm 0.78$ & $0.50 \pm 0.59$ & $<0.001$ \\
Emotional function & $0.76 \pm 0.66$ & $0.30 \pm 0.38$ & $<0.001$ \\
\hline
\end{tabular}

$* p<0.05 . R Q L Q$ : rhinoconjunctivitis quality of life, SCIT: subcutaneous immunotherapy

Table 3. Comparison of RQLQ scores in the SLIT group before and after receiving at least 1 year of immunotherapy

\begin{tabular}{lccc}
\hline \multicolumn{1}{c}{ RQLQ } & Before SLIT & After SLIT & p-value* \\
\hline Activity limitation & $1.84 \pm 1.09$ & $1.22 \pm 0.95$ & $<0.001$ \\
Sleep problems & $1.46 \pm 1.18$ & $0.90 \pm 1.03$ & $<0.001$ \\
Nose symptoms & $2.25 \pm 1.02$ & $1.44 \pm 0.92$ & $<0.001$ \\
Eye symptoms & $0.93 \pm 0.92$ & $0.71 \pm 0.79$ & 0.012 \\
Non-nose/eye symptoms & $1.22 \pm 0.93$ & $0.67 \pm 0.81$ & $<0.001$ \\
Practical problems & $0.90 \pm 1.04$ & $0.80 \pm 0.81$ & 0.002 \\
Emotional function & $0.91 \pm 0.87$ & $0.66 \pm 0.78$ & 0.004
\end{tabular}

*p<0.05. RQLQ: rhinoconjunctivitis quality of life, SLIT: sublingual immunotherapy

Table 4. Rate comparisons of the decreased RQLQ scores after receiving at least 1 year of immunotherapy between the 2 groups

\begin{tabular}{llcc}
\hline \multicolumn{1}{c}{ RQLQ } & SCIT (\%) & SLIT(\%) & p-value \\
\hline Activity limitation & 37.77 & 35.60 & 0.546 \\
Seep problems & 47.15 & 40.46 & 0.348 \\
Nose symptoms & 36.41 & 39.99 & 0.865 \\
Eye symptoms & 30.31 & 30.63 & 0.987 \\
Non-nose/eye symptoms & 32.11 & 20.51 & $0.015^{*}$ \\
Practical problems & 17.40 & 9.42 & 0.126 \\
Emotional function & 22.45 & 13.84 & 0.131 \\
\hline
\end{tabular}

*p<0.05. RQLQ: rhinoconjunctivitis quality of life, SCIT: subcutaneous immunotherapy, SLIT: sublingual immunotherapy

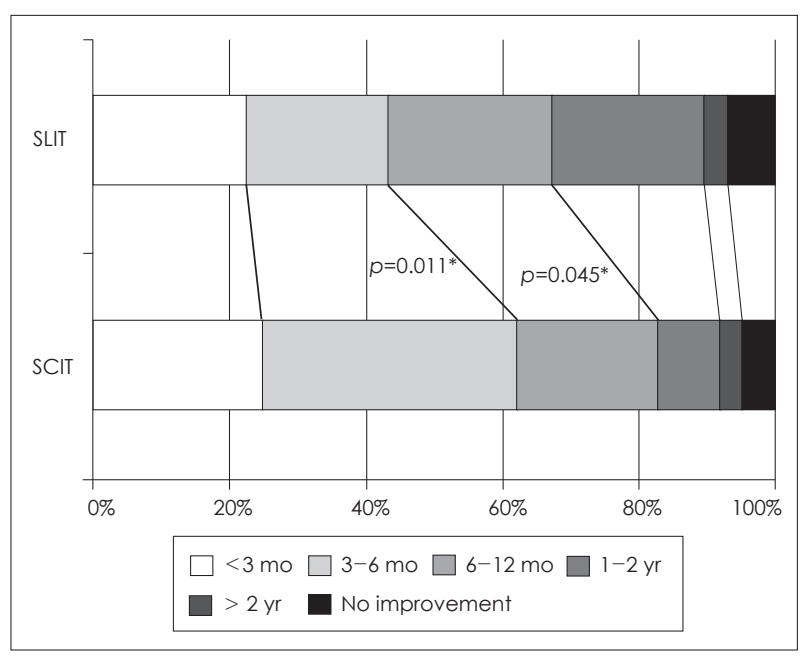

Fig. 1. Comparison of the time interval when the patients experienced symptom improvement for the first time after starting immunotherapy. ${ }^{*} p<0.05$. SCIT: subcutaneous immunotherapy, SLIT: sublingual immunotherapy. (gender, age, duration). RQLQ scores before immunotherapy between the two groups had no significant differences at all domains (Table 1).

After 1 year of immunotherapy, both the SCIT and SLIT groups had significantly improved ROLQ scores at all domains (Table 2 and 3).

When comparing the rate of improved RQLQ scores between SCIT and SLIT groups, SCIT improved the non-nose/ eye symptoms of the patients significantly more than SLIT (SCIT: $32.11 \%$ vs. SLIT: $20.51 \%$ ) (Table 4).

There were no differences between SCIT and SLIT groups in overall time interval when the patients experienced symptom improvement for the first time after starting immunotherapy ( $p=0.833$ ). However, when comparing clinical improvement at 6 months and 1 year, SCIT improved the symptoms faster than SLIT ( $p$-values $=0.011$ and 0.045 respectively) as shown in Fig. 1 . 
There were no differences between SCIT and SLIT in the frequency of hospital visits, physician visiting time or adverse effects ( $p=0.722,0.809$ and 0.902 , respectively). Additionally, there were no differences between SCIT and SLIT groups in medication use, healthcare costs or overall satisfaction ( $p=0.712$, 0.450 and 0.542, respectively) as shown in Fig. 2.

Among the patients, $89.3 \%$ in the SCIT group and $81.6 \%$ in the
SLIT group would continue immunotherapy. The dropout rates in the SCIT group were lower than in the SLIT group (13\% and $21 \%$, respectively) but without significant difference $(p=0.208)$.

\section{Discussion}

Immunotherapy aims to prevent the immunological reaction
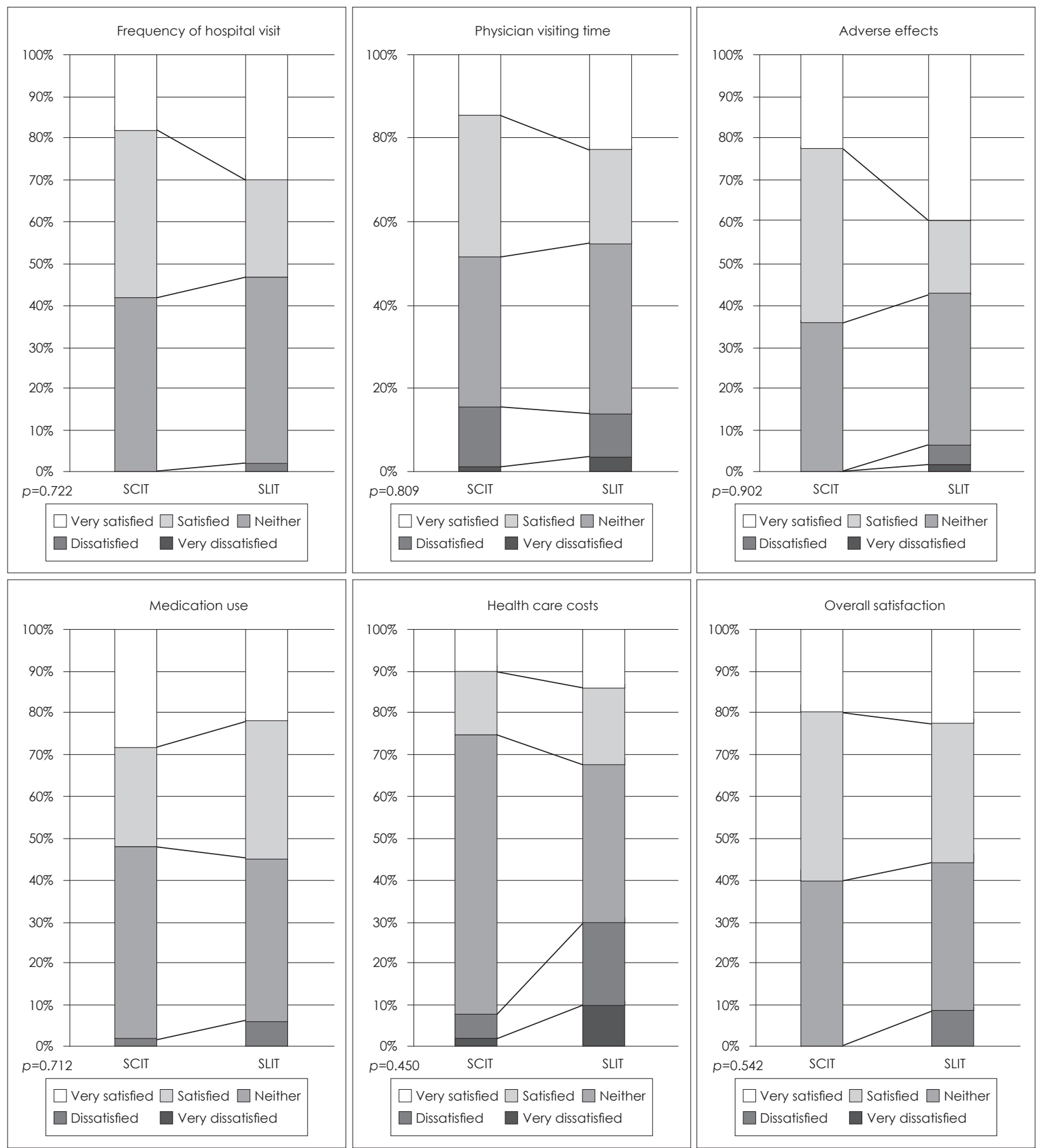

Fig. 2. Comparison of hospital visit frequency, physician visiting time, adverse effects, medication use, healthcare costs and overall satisfaction between SCIT and SLIT groups. SCIT: subcutaneous immunotherapy, SLIT: sublingual immunotherapy. 
of allergy sufferers to specific allergens. The current standard therapy is subcutaneous administration of clinically relevant allergens for several months. However, SCIT had several disadvantages, so SLIT was developed. ${ }^{3,4)}$

Many comparative clinical studies of SCIT and SLIT were reported after SLIT was performed. Khinchi, et al. ${ }^{9}$ founded the SCIT group had a disease severity reduced to one-third and in the SLIT group to half the severity observed in placebotreated patients. In contrast to several studies reported that SLIT and SCIT had the same efficacy and no differences in medical usage in the patients with allergic rhinitis to HDM who received immunotherapy for at least 6 months. ${ }^{10)}$ However, no studies have been conducted regarding SLIT and SCIT in Korea.

In present study, we compared clinical outcomes and satisfaction of patients allergic to HDMs after SCIT or SLIT. The symptom score index has not been able to express fully the states of the perennial allergic rhinitis patients. So, other tools were deemed necessary to not only evaluate the degree of severity but also to evaluate the quality of life on allergic rhinitis patients. RQLQ has been known that it was effective as an evaluator of impairment of quality of life in patients. ${ }^{12,13)}$ So that we used the RQLQ and a questionnaire on patient's satisfaction.

There were no statistically significant differences in gender, age, degree of sensitization to allergens, duration of treatment, symptom scores before immunotherapy including activity limitation, sleep problem, nose symptom, eye symptom, non-nose/eye symptoms, practical symptom or emotional function scores. Thus, the slight imbalances in demographic characteristics between the SCIT and SLIT groups were not statistically significant and did not affect the statistical results. When comparing the 2 groups, SCIT reduced the nonnose/eye symptoms in patients with allergic rhinitis significantly more than SLIT. This domain contains fatigue, thirst, reduced productivity, irritability, poor concentration, and headache. This result shows SCIT may effective to non-nose/eye symptoms. But more research is needed to verify these results, because our sample is small.

In patient satisfaction questionnaires such as hospital expenses, physician visiting time and side effects, there were no differences between the 2 groups. But more patients in the SCIT group had clinical improvement after 6 months and 12 months of immunotherapy indicating SCIT can improve allergic symptoms faster than SLIT. This result is thought to have affected that SCIT group showed a lower dropout rate. Similar result was studied by Chang, et al. ${ }^{11)}$ They studied the efficacy of at least 6 months of SLIT treatment in patients with allergic rhinitis to HDMs and found the subjective symptoms were improved with SLIT, but the dropout rate was high despite the symptomatic improvement. Although SLIT has similar effect to SCIT, this study shows SCIT is more effective in improving non-nose/eye symptoms and may relieve the symptoms faster than SLIT.

This study was performed in only one medical center and we evaluated patients by only questionnaires. So we plan to study using the medication score and physical change. Also, further study will be needed to find why SCIT is more effective to non-nose/eye symptoms and faster than SLIT.

\section{REFERENCES}

1) Akdis CA. Allergy and hypersensitivity: mechanisms of allergic disease. Curr Opin Immunol 2006;18(6):718-26.

2) Brozek JL, Bousquet J, Baena-Cagnani CE, Bonini S, Canonica GW, Casale TB, et al. Allergic Rhinitis and its Impact on Asthma (ARIA) guidelines: 2010 revision. J Allergy Clin Immunol 2010;126 (3):466-76,

3) Akdis CA, Akdis M. Mechanisms of allergen-specific immunotherapy. J Allergy Clin Immunol 2011;127(1):18-27; quiz 28-9.

4) Casale TB, Stokes JR. Immunotherapy: what lies beyond. J Allergy Clin Immunol 2014;133(3):612-9; quiz 620.

5) Calderón MA, Simons FE, Malling HJ, Lockey RF, Moingeon $P$, Demoly P. Sublingual allergen immunotherapy: mode of action and its relationship with the safety profile. Allergy 2012;67(3):302-11.

6) Kleine-Tebbe J, Ribel M, Herold DA. Safety of a SQ-standardised grass allergen tablet for sublingual immunotherapy: a randomized, placebo-controlled trial. Allergy 2006;61(2):181-4.

7) Moingeon P, Batard T, Fadel R, Frati F, Sieber J, Van Overtvelt L. Immune mechanisms of allergen-specific sublingual immunotherapy. Allergy 2006;61(2):151-65.

8) Quirino T, Iemoli E, Siciliani E, Parmiani S, Milazzo F. Sublingual versus injective immunotherapy in grass pollen allergic patients: a double blind (double dummy) study. Clin Exp Allergy 1996;26(11): 1253-61.

9) Khinchi MS, Poulsen LK, Carat F, André C, Hansen AB, Malling HJ. Clinical efficacy of sublingual and subcutaneous birch pollen allergen-specific immunotherapy: a randomized, placebo-controlled, double-blind, double-dummy study. Allergy 2004;59(1):45-53.

10) Saporta D. Efficacy of sublingual immunotherapy versus subcutaneous injection immunotherapy in allergic patients. J Environ Public Health 2012;2012:492405.

11) Chang H, Han DH, Mo JH, Kim JW, Kim DY, Lee CH, et, al. Early compliance and efficacy of sublingual immunotherapy in patients with allergic rhinitis for house dust mites. Clin Exp Otorhinolaryngol 2009;2(3):136-40.

12) Juniper EF, Thompson AK, Ferrie PJ, Roberts JN. Validation of the standardized version of the Rhinoconjunctivitis Quality of Life Questionnaire. J Allergy Clin Immunol 1999;104(2 Pt 1):364-9.

13) Park KH, Cho JS, Lee KH, Shin SY, Moon JH, Cha CI. Rhinoconjunctivitis Quality of Life Questionnaire (RQLQ) as an evaluator of perennial allergic rhinitis patients-the first report-. Korean J Otolaryngol-Head Neck Surg 2002;45(3):254-62. 